\title{
ELABORACIÓN DE INSTRUMENTOS (ESCALAS DE VALORACIÓN Y GRADUADAS) PARA LA EVALUACIÓN FORMATIVA
}

Development of tools (assessment and graduated scales) for the formative assessment Desenvolvimento de instrumentos (escalas de valorização e graduadas) para a avaliação formativa

Ángel Pérez-Pueyo (1)

Mario Sobejano Carrocera (2)

Universidad de León, España. Teléfono: +34 619867767. E-mail: angel.perez.pueyo@unileon.es

(2) IES Nueve Valles. Puente San Miguel, Cantabria. Teléfono: +34 620920886. Email: mario.sobejano@gmail.com

\section{Resumen}

Los instrumentos de evaluación se han convertido en uno de los elementos claves para la incorporación del alumnado al proceso de aprendizaje activo. El taller pretende comprobar la utilidad de las escalas complejas de carácter descriptivo entre las que se encuentran las Rúbricas, las Escalas de valoración y las Escalas graduadas. Estos tres tipos de instrumentos son consecuencia de la evolución en el trabajo de los docentes por ofrecer al alumnado un instrumento que proporcione un feedback lo más objetivo y ajustado posible a la valoración de la producción. La intención es ayudar al alumnado en el proceso de aprendizaje cuando estos son utilizados en la evaluación formativa.

Palabras clave: Instrumentos de evaluación; rúbrica; escala de valoración; escala graduada

\begin{abstract}
The assessment tools have become one of the key elements for the incorporation of students in the process of active learning. The workshop aims to test the usefulness of descriptive complex scales including rubrics, rating scales and graduated scales. These three types of instruments are a consequence of the evolution in the work of the teachers to offer the students an instrument that provides feedback as close and objective as
\end{abstract}


possible to the assessment of a production. The intention is to help students in the learning process when they are used in formative assessment.

Keywords: Assessment tools; rubric; assessment scale; graduated scale

\section{Resumo}

As ferramentas de avaliação tornaram-se um dos elementos-chave para a incorporação de estudantes para processo de aprendizagem ativo. O workshop visa testar a utilidade das escalas descritivas complexas entre os quais estão as rubricas, escalas de valorização e escalas graduadas. Estes três tipos de instrumentos são o resultado da evolução do trabalho dos professores para oferecer aos alunos um instrumento que fornece feedback e ajustado o mais objetivo possível a avaliação da produção. A intenção é ajudar os alunos no processo de aprendizagem quando eles são usados na avaliação formativa.

Palavras-chave: Ferramentas de avaliação; rubricas; escalas de valorização; escalas graduadas

\section{Los instrumentos de evaluación: elemento clave en la evaluación formativa}

Desde 1990, la terminología sobre evaluación ha evolucionado notablemente aunque este mismo hecho ha generado una significativa incoherencia en relación a su concreción y utilización tanto en la educación no universitaria como en los estudios universitarios (López-Pastor \& Pérez-Pueyo, 2017; Pérez-Pueyo, Hortigüela \& Gutiérrez-García, 2017).

López-Pastor y Pérez-Pueyo definen los Instrumentos de evaluación como “aquellos documentos o recursos que se vinculan a una actividad de evaluación, para establecer en ellos los requisitos y los aspectos a valorar, indicando con claridad los niveles de logro de cada uno” (2017, p. 80). De hecho, esta definición es corroborada por algunas comunidades autónomas como Castilla y León que determinan que son "todos aquellos documentos o registros utilizados por el profesorado para la observación sistemática y el seguimiento del proceso de aprendizaje del alumno" (Orden EDU/888/2009, art. 3.1). 
Estos instrumentos, utilizados desde una perspectiva formativa, nos permiten mejorar los procesos de enseñanza y aprendizaje y como comenta López-Pastor, “sirven para que el alumnado aprenda más (y/o corrija sus errores) y para que el profesorado aprenda a trabajar mejor (a perfeccionar su práctica docente)” (LópezPastor et al., 2006, p. 36), adentrándonos en la Evaluación formativa, sobre la que la evidencia científica ha demostrado las enormes ventajas para la mejora del aprendizaje del alumnado (Biggs, 2005; Black \& Williams, 1998; Boud, 1995; Boud \& Falchikov, 2007; Dochy, Segers \& Dierick, 2002; López-Pastor, 1999, 2006, 2013).

Desde el inicio del siglo XXI, con la implantación del Espacio Europeo de Educación Superior y el concepto de competencia, las rúbricas han aparecido en el panorama educativo como un instrumento habitual para la evaluación de éstas.

\section{Contextualización del taller: Educación Universitaria y no Universitaria}

Tanto en el ámbito universitario como en el no universitario, las rúbricas se han convertido en un instrumento cotidiano. Sin embargo, no siempre son todo lo útiles que se espera de ellas al no detallar en algunos casos los niveles de logro necesarios para que el alumnado pueda aprovecharlos adecuadamente; pero, sobre todo, cuando se vinculan a los procesos de calificación.

De hecho, en los últimos años, cuando se han comenzado a desarrollar procesos triádicos de evaluación (Domínguez-Fernández, 2015; Pérez-Pueyo, 2017) en los que el mismo instrumento se utiliza en procesos de autoevaluación, coevaluación y heteroevalaución, la utilización de instrumentos con un mayor nivel descriptivo como las Escalas de valoración o las Escalas graduadas están proporcionando mejores resultados por la coincidencia de las valoraciones. Además, esta mayor concreción de los instrumentos está ofreciendo un mejor ajuste de las valoraciones.

\section{Descripción del taller}

La pretensión del taller es identificar con claridad tres tipos de instrumentos: las Rúbricas, las Escalas de Valoración y las Escalas Graduadas; así como entender la evolución que han seguido dentro de las denominadas escalas complejas de carácter descriptivo. 
Comenzamos con la denominada escala descriptiva básica, conocida como Rúbrica. Este término procede del contexto educativo anglosajón en el que denominan “rubric” a lo que en España hemos llamado tradicionalmente "escalas descriptivas”. Éstas son matrices o escalas que identifican una serie de aspectos a valorar que, normalmente en 3 ó 4 niveles de logro, describen las características que posee una producción que se pretende valorar con precisión. Sin embargo, no todas las matrices que se organizan con esta forma deberían ser consideradas rúbricas (escalas descriptivas) pues, en muchas ocasiones no son más que escalas verbales. Generalmente, en estos instrumentos, con un mismo texto descriptivo, sólo suele cambiar un adverbio de frecuencia para determinar la diferencia el nivel (por ejemplo: siempre, casi siempre, casi nunca, nunca) (Pérez-Pueyo, \& López-Pástor, 2017).

Aunque las rúbricas suelen identificar los diferentes niveles con valores cualitativos, cuando se pretenden utilizar para calificar se les tiende a dar valores numéricos o establecer horquillas de nota en cada nivel, incluso asignando porcentajes a los aspectos a valorar, pasando a denominarse Rúbricas de puntuación.

Cuando las rúbricas se van retorciendo y los aspectos a valorar tienen diferente número de niveles de logro, valor relativo diferente e, incluso, cambia la forma de presentación, nos adentramos en las denominadas Escalas de valoración diferenciada. La característica fundamental de este instrumento es que su nivel de concreción y de diferenciación en los distintos aspectos a valorar es mucho más detallada y útil en el proceso de aprendizaje.

Por ello, tanto las Rúbricas de puntuación como las Escalas de valoración se caracterizan por tener un enfoque analítico que establece que la calificación sea numéricamente exacta. Sin embargo, lo que inicialmente puede parecer ideal, cuando las producciones tienen un componente creativo apenas ofrecen al docente margen de maniobra en la valoración. En este caso es tremendamente difícil describir los niveles por anticipado y cuantificar el valor en el resultado final de la producción. Es por ello que surge la evolución de los dos instrumentos anteriores hacia las denominadas Escalas Graduadas. 


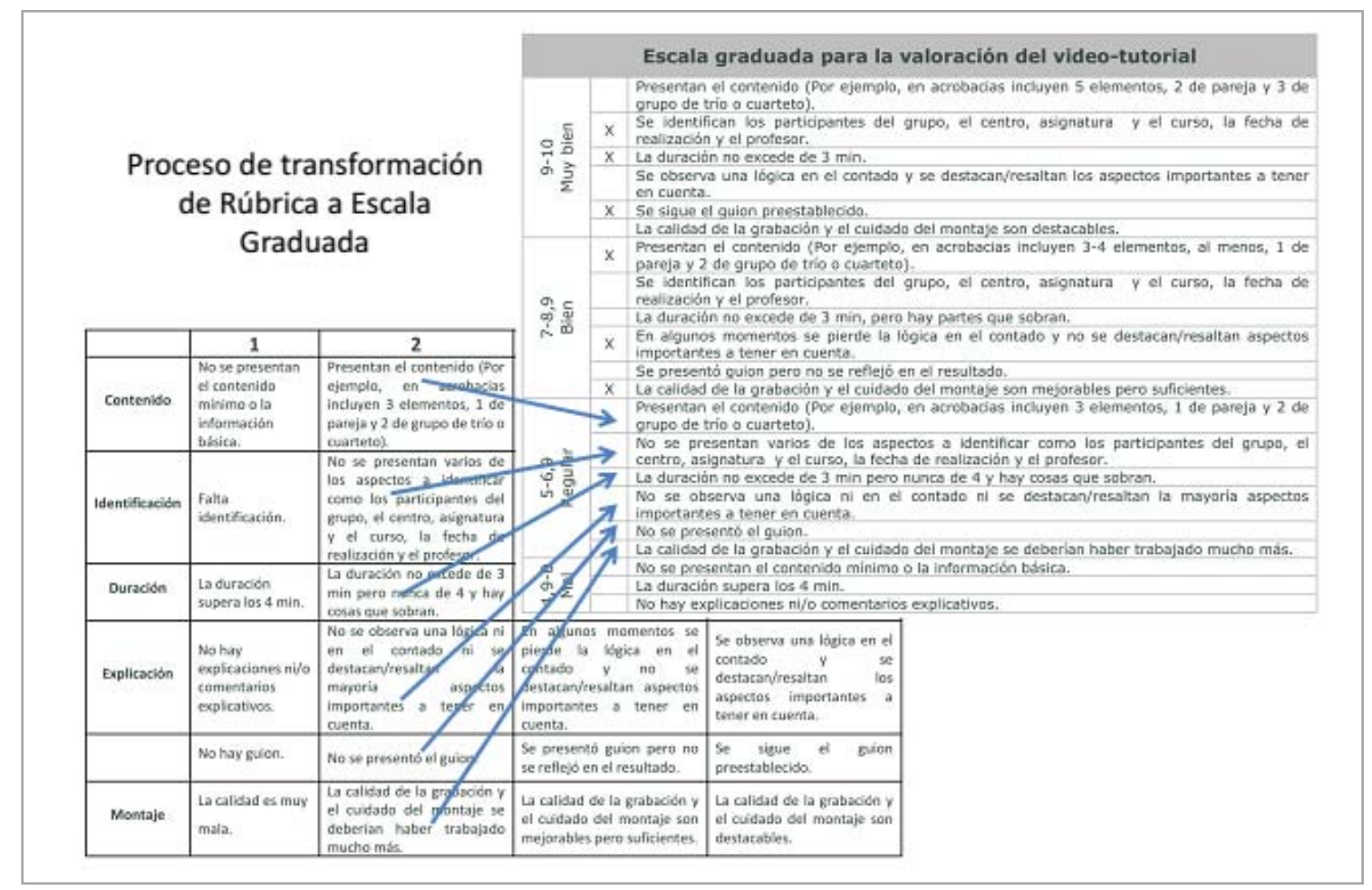

Figura 1.

Trasformación de Rúbrica a Escala graduada. Elaboración propia.

En éstas se organizan los niveles de logro por horquillas, permitiendo valorar en éstas los aspectos más formales, y dejar a la discrecionalidad técnica ${ }^{1}$ del docente la posibilidad de establecer diferencias entre las producciones concretando la nota dentro de la horquilla sin perder un razonable grado de objetividad.

\section{Reflexiones finales}

El taller espera ofrecer a los docentes la posibilidad de elegir entre los instrumentos más adecuados, dentro de las escalas que describen los niveles de logro de alumnado, en función de las producciones solicitadas. De este modo, involucrar a los alumnos en el proceso de aprendizaje permitirá desarrollar evaluaciones triádicas en las que se cotejen las valoraciones desde los diferentes agentes implicados.

\section{Referencias}

Biggs, J. (2005). Calidad del aprendizaje universitario. Madrid: Narcea.

\footnotetext{
${ }^{1}$ La discrecionalidad técnica es el concepto que los juristas utilizan para aceptar la opinión de los tribunales tras haber establecido éstos previamente unos criterios básicos en horquillas de las valoraciones de las pruebas a evaluar. A estos criterios en horquillas les llaman aledaños.

Elaboración de instrumentos (escalas de valoración y graduadas) para la evaluación formativa
} 
Black, P. \& Williams, D. (1998). Assessment and classroom learning. Assessment in Education, 4(1), 7-71.

Boud, D. (1995). Enhancing Learning Through Self-Assessment. London: Routledge.

Boud, D. \& Falchikov, N. (2007). Rethinking Assessment in Higher Education. Learning for the long term. Oxon: Routledge.

Dochy, F., Segers, M., \& Dierick, S. (2002). Nuevas vías de aprendizaje y enseñanza y sus consecuencias: una era de evaluación. Revista de Docencia Universitaria, 2(2), 13-30.

Domínguez-Fernández, R., González-Alonso, M. I., Fernández-Fernández, R., PérezPueyo, A., Gutiérrez García, C., Alonso-Cortés Fradejas, M. D., Diez-Fernández, A., \& García-González, M. E. (2015). Propuesta para una doble evaluación triádica del Máster de Educación Secundaria. En A. Fidalgo Blanco, M. L. SeinEchaluce Lacleta, \& F. J. García- Peñalvo (Coords.). La sociedad del Aprendizaje. Actas del III Congreso internacional sobre Aprendizaje Innovación y Competitividad (pp. 565-570. Madrid: Fundación General de la Universidad Politécnica de Madrid.

López-Pastor, V. M. (1999) Prácticas de evaluación en Educación Física: estudio de casos en Primaria, Secundaria y Formación del Profesorado. Valladolid: Universidad de Valladolid.

López-Pastor, V.M. (Coord.) (2006). La Evaluación en Educación Física. Revisión de los modelos tradicionales y planteamiento de una alternativa: la evaluación formativa y compartida. Buenos Aires: Miñó y Dávila

López-Pastor, V. M. (2013). Evaluación en Educación Física. Revisión internacional de la temática. Revista de Educación Física. Renovar la teoría y la práctica, 29(3), 4-13.

López-Pastor, V. M. \& Pérez-Pueyo, A. (Coords.) (2017). Evaluación formativa y compartida en educación: experiencias de éxito en todas las etapas educativas. León: Universidad de León. Recuperado de https://buleria.unileon.es/handle/10612/5999 
Orden EDU/888/2009, de 20 de abril, por la que se regula el procedimiento para garantizar el derecho del alumnado que cursa enseñanzas de educación secundaria obligatoria y de bachillerato, en centros docentes de la Comunidad de Castilla y León, a que su dedicación, esfuerzo y rendimiento sean valorados y reconocidos con objetividad (B.O.C.y.L, 27 de abril).

Pérez-Pueyo, A. (2017). El estilo actitudinal como propuesta metodológica vinculada a la evaluación formativa. En V. M. López-Pastor \& Pérez-Pueyo (coords) Evaluación formativa y compartida en educación: experiencias de éxito en todas las etapas educativas (pp. 240-259). León: Universidad de León.

Pérez-Pueyo, A., Hortigüela, D., \& Gutiérrez-García, C. (2017). Reflexión sobre la evaluación en la formación inicial del profesorado en España. En búsqueda de la concordancia entre dos mundos. Revista Infancia, Educación y Aprendizaje, 2(2), 39-75.

Pérez-Pueyo, A., \& López-Pastor, V. M. (2017). Aclaración de los términos implicados en el proceso de evaluación educativa. En V. M. López-Pastor \& Pérez-Pueyo (coords) Evaluación formativa y compartida en educación: experiencias de éxito en todas las etapas educativas (pp. 70-91). León: Universidad de León. 